\title{
The sudden collapse of pollinator communities
}

J. Jelle Lever ${ }^{1,2 *}$ Egbert H. van $\mathrm{Nes}^{1}$, Marten Scheffer ${ }^{1}$, and Jordi Bascompte ${ }^{2}$

${ }^{1}$ Department of Aquatic Ecology and Water Quality Management, Wageningen University, P.O. Box 47, NL-6700 AA, Wageningen, The Netherlands

${ }^{2}$ Integrative Ecology Group, Estación Biológica de Doñana, CSIC

Calle Américo Vespucio s/n, 41092 Sevilla, Spain

\section{Submission of revised manuscript to Ecology Letters \#2}

E-mail addresses (in order of authorship):

jelle.lever@wur.nl, egbert.vannes@wur.nl, marten.scheffer@wur.nl, bascompte@ebd.csic.es

Authorship statement:

Simulations and data analysis were performed by Jelle Lever. A first draft of the manuscript was prepared by Jelle Lever. All authors contributed to the design of the research, discussed data, and contributed to writing the final manuscript.

Short running title:

The collapse of pollinator communities

Keywords:

pollinator decline, critical transitions, hysteresis, mutualistic networks, nestedness

Number of words:

Abstract: 144, Main text: 4700-4900, Caption of figure 1: 40, Caption of figure 2: 69, Caption of figure 3: 78, Caption of figure 4: 71, Caption of figure 5: 81, Figure 6: 147

Number of references: 50

Number of figures in the main text: 6

Type of article: Letter

Manuscript number: ELE-00853-2013

${ }^{*}$ To whom correspondence should be addressed. E-mail: jelle.lever@wur.nl, Phone: +31 647238146, Fax: +31 317419000 
2 Declines in pollinator populations may harm biodiversity and agricultural productivity. Little attention 3 has, however, been paid to the systemic response of mutualistic communities to global environmental 4 change. By using a modelling approach and merging network theory with theory on critical transitions, 5 we show that the scale and nature of critical transitions is likely to be influenced by the architecture 6 of mutualistic networks. Specifically, we show that pollinator populations may collapse suddenly once 7 drivers of pollinator decline reach a critical point. A high connectance and/or nestedness of the mutualistic 8 network increases the capacity of pollinator populations to persist under harsh conditions. However, once 9 a tipping point is reached, pollinator populations collapse simultaneously. Recovering from this single 10 community-wide collapse requires a relatively large improvement of conditions. These findings may have 11 large implications for our view on the sustainability of pollinator communities and the services they 12 provide. 


\section{INTRODUCTION}

Widespread declines in wild and domesticated pollinator populations raise concerns about the future of biodiversity and agricultural productivity (Allen-Wardell et al. 1998; Diaz et al. 2005; Biesmeijer et al. 2006; Potts et al. 2010; Burkle et al. 2013; Garibaldi et al. 2013). The majority of flowering plants depend on animals for pollination. Those plants are in turn at the basis of food webs and provide food for livestock and human populations (Klein et al. 2007; Ollerton et al. 2011). Pollinators thus provide an essential service to ecosystems and humanity. Assessing the potential for further degradation of this service is therefore of great importance.

A considerable effort is being made to identify the potential causes of declining pollinator abundances. Recently, field experiments showed how commonly used insecticides strongly increase pollinator mortality (Henry et al. 2012; Whitehorn et al. 2012). Habitat destruction, parasites, and disease are also seen as important drivers of pollinator decline. Most likely, a mix of those causes increases the mortality of pollinator populations (Diaz et al. 2005; Potts et al. 2010; Bryden et al. 2013).

The impact of a further increase in drivers of pollinator decline will depend strongly on the capacity of plant-pollinator communities to withstand a further increase in those drivers. Determination of the response of natural communities to environmental change is however notably hard, primarily because the response of these relatively complex systems depends on more than the intrinsic properties of species. A central role is likely to be played by the strength, number, and nature of interactions between species, and the way in which those interactions are arranged in ecological networks (May 1972; McCann 2000; Bascompte et al. 2006; May 2006; Ives \& Carpenter 2007; Scheffer et al. 2012). When assessing the impact of a further increase in the drivers of pollinator decline, it is thus of fundamental importance to take the topology of mutualistic networks (i.e., the number and way in which mutualistic interactions are arranged) into account.

Mutualistic networks, such as those made out of the interactions between plants and pollinators, are known to display a high degree of nestedness, i.e., the more specialist species tend to interact with a subset of the species where more generalist species interact with (see figure 1; Bascompte et al. 2003; Bascompte \& Jordano 2007). Theoretical work has shown that the nestedness of mutualistic networks increases the robustness of plant-pollinator communities to species extinctions (Memmott et al. 2004; Burgos et al. 2007) and habitat loss (Fortuna \& Bascompte 2006), the proportion of coexisting species once an equilibrium is reached (Bastolla et al. 2009; Thébault \& Fontaine 2010), and the speed at which the community returns to equilibrium after a perturbation (Okuyama \& Holland 2008; Thébault \& Fontaine 2010). 
Little attention, however, is given to the influence of mutualistic network topology on potential critical transitions in the size of pollinator populations. Ecosystems may respond in various ways to changing environmental conditions, such as the change in conditions caused by a further increase in drivers of pollinator decline, which may have profound implications for their resilience to environmental change (Scheffer et al. 2001; Scheffer \& Carpenter 2003). When conditions change gradually, the state of some systems (e.g., the size of populations) may change likewise, in a smooth, gradual manner. Other systems may respond strongly to change within a narrow range of environmental conditions, but are relatively insensitive to change outside of this range. Particularly sudden shifts may occur when a system has more than one stable state. Such a system cannot change smoothly from a one stable state (e.g., large population sizes) to an alternative stable state (e.g., small population sizes). Instead, a sudden shift occurs when environmental conditions pass a critical point. We refer to such shifts as 'critical transitions'. To return back to the original state after a critical transition, a return to conditions prior to the transition is often not sufficient; instead, a larger change in conditions is needed until another critical point is reached at which the system shifts back to the original state. The existence of a difference between the critical conditions at which a forward and backward transition occurs, is known as 'hysteresis'.

The notion that alternative stable states exist is supported by observations in a wide variety of ecological and experimental systems (Scheffer et al. 2001; Scheffer \& Carpenter 2003; Rietkerk et al. 2004; Kefi et al. 2007; Drake \& Griffen 2010; Veraart et al. 2011; Hirota et al. 2011; Dai et al. 2012). The complexity of many natural communities has however made it hard to develop the existing theory on alternative stable states further into a framework that helps us to assess their resilience (Scheffer et al. 2012). Here, we try to contribute to the development of such a framework, by merging theory on alternative stable states with theory on the structure of ecological networks. Specifically, we do this by examining the potential occurrence of critical transitions in the size of pollinator populations due to a change in a driver of pollinator decline. Subsequently, we study the way in which the connectance and nestedness of mutualistic networks may affect the community-wide implications of these shifts between alternative stable states. This will be done with the help of a mathematical model. 


\section{METHODS}

Nestedness algorithm. Networks with a different degree of nestedness were generated by using an algorithm similar to the one described by Medan et al. (2007). This algorithm was shown to generate networks that are similar to empirically studied plant-pollinator networks (also by Medan et al. 2007). The algorithm allows us to vary nestedness of networks with a given number of species, connectance and fraction of "forbidden links". Connectance is the fraction of all possible interactions that is occurring in the network. Forbidden links are interactions that cannot occur, for example because of a morphological or phenological uncoupling (e.g., between late-flowering plant species and early seasonal pollinator species, see Jordano et al. 2003).

Initially, the algorithm assigns with a predefined probability mutualistic interactions and forbidden links between two species groups. This results in a network with a random structure, of which the probability of having an interaction corresponds to the connectance of the network and the probability of a forbidden link to the fraction of forbidden links. In case any of the species has no interactions, a new randomly structured network is generated.

In order to generate nested networks, interactions are re-arranged within the network. During each iteration the algorithm randomly selects an interaction between two species $a$ and $b$. This interaction is changed into an interaction between species $a$ and randomly selected species $c$, when this species has more interactions than species $b$. During the iterative process, species thus start to interact more with species that already have many interactions. This "rich get richer" mechanism increases the nestedness of the network. Iterations are continued until a desired nestedness is reached.

Two exceptions to the above mentioned rule exist. The interaction is not changed from an interaction with species $b$ to an interaction with species $c$, when species $b$ has only one interaction, or when the interaction between species $a$ and $c$ is forbidden. This ensures that each species remains having at least one interaction, and that the identity of forbidden links is not changed by the algorithm.

We derive the nestedness of the entire network, $N$, as in Bastolla et al. (2009):

$$
N=\frac{\sum_{i<j}^{P} N_{i j}+\sum_{i<j}^{A} N_{i j}}{\frac{S_{P}\left(S_{P}-1\right)}{2}+\frac{S_{A}\left(S_{A}-1\right)}{2}},
$$

where $S_{P}$ is the number of plant species, $S_{A}$ is the number of pollinator species and $N_{i j}$ is the nestedness of species pair $i$ and $j$, which is derived as follows:

$$
N_{i j}=\frac{n_{i j}}{\min \left(n_{i}, n_{j}\right)}
$$

98 where $n_{i j}$ is the number of times species $i$ and $j$ interact with the same mutualistic partner, $n_{i}$ is the number of interactions of species $i$ and $n_{j}$ is the number of interactions of species $j$. 
All networks generated with the procedure above were checked for the potential presence of more than one component (i.e., a group of species that is completely disconnected from the rest of the network). If more than one component was found, the network was dismissed from our analysis, and replaced with a newly generated network, consisting of only one component.

Model of mutualistically interacting species. In an attempt to disentangle the relationship between network structure and the response of plant-pollinator communities to environmental change, we studied the impact of mutualistic network topology on the behaviour of a dynamic model. Our dynamic model describes two mutualistically interacting species groups; plants and pollinators. Species belonging to the same group are in direct competition with each other, while mutualistic interactions occur between species belonging to a different group. The pollinators are subjected to a gradual change in mortality and/or growth rate, caused by a change in one of the drivers of pollinator decline.

The model, describing a group of $S_{P}$ plant species and $S_{A}$ pollinator species, is as follows:

$$
\begin{aligned}
& \frac{d P_{i}}{d t}=r_{i} P_{i}+\frac{\sum_{k=1}^{S_{A}} \gamma_{k i} A_{k}}{1+h_{i} \sum_{k=1}^{S_{A}} \gamma_{k i} A_{k}} P_{i}-\sum_{j=1}^{S_{P}} C_{i j} P_{j} P_{i}+\mu_{P}, \\
& \frac{d A_{k}}{d t}=\left(r_{k}-d_{A}\right) A_{k}+\frac{\sum_{i=1}^{S_{P}} \gamma_{i k} P_{i}}{1+h_{k} \sum_{i=1}^{S_{P}} \gamma_{i k} P_{i}} A_{k}-\sum_{l=1}^{S_{A}} C_{k l} A_{k} A_{l}+\mu_{A},
\end{aligned}
$$

where $P_{i}$ represents the abundance of plant species $i$ and $A_{k}$ represents the abundance of pollinator species $k$. Intrinsic growth rates, i.e., the growth independent from mutualistic and competitive interactions, are represented by $r$, which is species-specific and can either be positive or negative. A general reduction of pollinator growth rates or increase in pollinator mortality rates, affecting all pollinator species, is included with driver of pollinator decline, $d_{A}$.

Population growth is enhanced by mutualistic partners (i.e. the pollinator or plant species providing a service or resource to the plant or pollinator population). Like Okuyama \& Holland (2008) and Bastolla et al. (2009), we assume that the beneficial effect of mutualistic partners on population growth saturates when the abundance of mutualistic partners is high. The extent of this saturation is determined by halfsaturation constants $h$. We assume mutualistic interactions to be either absent, in which case mutualistic interaction strength, $\gamma$, is equal to zero, or to be present, in which case the mutualistic interaction strength is assumed to depend on the degree of the node benefiting from the interaction in the following manner:

$$
\gamma_{m n}={\frac{\gamma_{0}}{K_{n}}}^{t},
$$


in which, for each interaction, $\gamma_{0}$ is taken from a uniform distribution, $K_{n}$ is the number of interactions of the species befitting from the interaction, and $t$ determines strength of the trade-off between interaction strength and number of interactions. Both $t=0$ (no trade-off) and $t=1$ (full trade-off), represent "neutral" cases. Assuming no trade-off is neutral in the sense that the strength of mutualistic interactions is not changed by the topology of the network, while a full trade-off assumes that the gain species have from their mutualistic interactions is not changed by the topology of the network. Ecological reality is likely to lie somewhere in between those two extremes. The strength of competition between individuals of the same species group is determined by $C$. We study a system where species do not outcompete each other when mutualistic partners are absent (as in Van Nes \& Scheffer 2004). Intraspecific competition, $C_{i i}$, is therefore assumed to be substantially stronger than interspecific competition $C_{i j}$. Lastly, a small immigration factor $\mu$ is incorporated in order to allow for the (re-)establishment of otherwise extinct species. $\mu$ is not supposed to influence the dynamics of the model.

Simulations and parameter settings. We examined the response of pollinator populations to increasingly harsh conditions, by gradually increasing the driver of pollinator decline, $d_{A}$. This gradual increase was simulated by a stepwise increase in the driver of pollinator decline, with step size 0.01 . For each step, we ran our model until equilibrium was reached, by applying a Runge-Kutta method that numerically solves our model. We increased the driver of pollinator decline past the point where all pollinator species are extinct (i.e., have an abundance lower than 0.01). After this point was reached, we simulated improving conditions by gradually decreasing the driver of pollinator decline, again with a step size of 0.01 . This allowed us to check for hysteresis.

We scanned for the occurrence of sudden changes in pollinator abundance within a small range of change in the driver of pollinator decline. We defined a "sudden change" as a change in pollinator abundance that was larger than 0.2 over an in- or decrease in the driver of pollinator decline of 0.01 (one step in our simulations). This allowed us to differentiate between a sudden and a gradual extinction or recovery of pollinator populations.

In our default approach, we made simulations for communities consisting out of 25 plants and 25 pollinator species. The impact of connectance on the behaviour of the model was tested by varying the connectance of communities with a random network topology. The impact of nestedness was studied by comparing networks differing in nestedness, but equal in connectance $(\mathrm{D}=0.15)$ and fraction of forbidden links $(\mathrm{F}=0.3)$. We, however, made sure that the qualitative behaviour of our model does not depend on a specific number of species, connectance or fraction of forbidden links chosen (see supplementary material 3). For each level of connectance and nestedness, we tested 250 different networks created with the above algorithm.

Unless stated otherwise, parameters were sampled from the following uniform distributions: $r_{i} \sim$ 
$U(0.05,0.35), \gamma_{0, i k} \sim U(0.8,1.2), h_{i} \sim U(0.15,0.3), C_{i i} \sim U(0.8,1.1), C_{i j} \sim U(0.01,0.05)$, or given the following value: $t=0.5, \mu=0.0001$.

The feasibility of networks. In order to allow for partial collapses of the plant-pollinator community, a substantial variation in growth rate, competition, and mutualistic interaction strength is needed. As a result of this variation, we did not always find a feasible solution, where the abundances of all species were higher than 0.01. If no feasible solution was found for a certain network, parameters were re-sampled until a feasible solution was found. If after 500 attempts no solution was found the network was discarded as non-feasible.

The net effect of species on each other. Net-relationships between pollinators were studied by numerically determining the influence of a small change in growth rate of species $l$ on the abundance of species $k\left(d A_{k} / d r_{l}\right)$. If an increase in growth rate of species $l$ leads to an increased abundance of species $k$, the net effect of species $l$ on species $k$ is positive (following Stone \& Roberts 1991). 
The majority of pollinator populations collapse suddenly to extinction once the driver of pollinator decline, $d_{A}$, reaches a critical value. These sudden collapses occur due to a positive feedback mechanism that results from the positive interactions between plants and pollinators. A large pollinator population size enhances the growth and thus the population size of plants, which in turn enhances the growth of the pollinator populations. As the strength of the driver pollinator decline, $d_{A}$, increases, this positive feedback mechanism maintains pollinator populations under conditions where they cannot recover from extinction (see supplementary material 1). Under these conditions, multiple alternative stable states may therefore exist, varying from a state where all pollinator populations are present to a state where some or all pollinator species are extinct. As the strength of the driver of pollinator decline, $d_{A}$, increases further, a critical point is reached where the strength of this feedback mechanism is no longer sufficient to maintain pollinator populations. At this point a critical transition occurs, leading to the sudden collapse of some or all pollinator populations. In communities with a random network topology and a relatively low connectance, we typically observe several partial collapses involving the extinction of few species. Nested communities with an equal connectance, however, tend to exhibit only one point of collapse, involving the extinction of the entire community (see figure 2).

Once the driver of pollinator decline has increased beyond the point where all pollinator populations have collapsed, a small decrease in mortality rates may not be sufficient for species to recover. As was the case with the sudden collapses, observed when the driver of pollinator decline, $d_{A}$, was increased, pollinator populations may also recover suddenly when the driver of pollinator decline is decreased. Especially in nested communities, the difference between the first point of recovery and the final point of collapse can be substantial when compared to randomly structured communities (see figure 3). A considerable improvement of conditions might thus be necessary before species can recover from collapse, which is indicative of hysteresis.

Multiple points of recovery were typically observed within communities that also exhibited several network collapses. In randomly structured communities, with a connectance of 0.15 , for example, multiple points of sudden recovery were found in $92 \%$ of the feasible communities in which also multiple collapses were observed. More than one sudden recovery was however only observed in $21 \%$ of the feasible communities that exhibited one point of collapse.

The ranking of species recovery was, in most feasible communities, similar to the order in which they collapsed. E.g., the species who were the last to collapse when the driver of pollinator decline, $d_{A}$, was increased, always recovered before or simultaneously with species that collapsed at a lower value of pollinator decline, in $79 \%$ of randomly structured communities with a connectance of 0.15 .

Further, sudden changes in the pollinator community always coincided with sudden changes in the plant community (see supplementary material 2). 
The potential for a single community-wide collapse. The probability of having a single community-wide collapse, instead of having several partial collapses, is strongly influenced by the connectance and/or nestedness of mutualistic networks. The fraction of networks, equal in connectance and nestedness, in which a single community-wide collapse was observed, can be seen as a measure of this probability.

The left panel of figure 4 shows the impact of connectance on the number of collapses that occur when the driver of pollinator decline, $d_{A}$, is increased. As the connectance of randomly structured communities increases, the fraction of communities that exhibit only one single point of community-wide collapse grows, until eventually almost no partial collapses are observed.

In the right panel of figure 4 , we show what happens when the nestedness of communities with a connectance of 0.15 is increased. A small increase in nestedness from 0.2 to 0.25 is already sufficient to observe a substantial decrease in the occurrence of partial collapses. When nestedness is increased further, almost no partial collapses are observed any more. Consequently, by increasing the nestedness, we thus observe a strong reduction in the occurrence of partial collapses, even though the connectance of those networks was fixed.

The cases where we did find a partial collapse in a highly nested community represent an extreme case where a large fraction of specialists interacts only with one single generalist. This generalist may, together with the specialists associated to it, collapse independent of the rest of a highly nested community.

As described in the Methods section, we needed a substantial variation in growth rate, competition and mutualistic interaction strength in order to allow for partial collapses of the plant-pollinator community. As a result of this variation, the parameters drawn from uniform distributions did not always give a feasible solution. A large fraction of randomly structured networks with a connectance of 0.15 , however, gave a feasible solution, and the majority of them also showed partial collapses. Surprisingly, the feasibility of networks was lowest for intermediate values of nestedness. Feasible solutions were thus most easily found in networks that where either fully random, or fully nested (see figure 4 and supplementary material 3 ). Networks for which it was hard to find a feasible solution, often had a small fraction of species that, during all attempts made to find a feasible solution, could not coexist with all others. Non-feasibility was thus almost always a property of this small fraction of species, rather than a property of the community as a whole. 
Pollinator persistence under changing environmental conditions. Network topology influences not only the probability of a single community-wide collapse; it is also important for the capacity of pollinator communities to persist under increasingly harsh conditions. Here, we measure this capacity as the amount of increase in the driver of pollinator decline, $d_{A}$, needed to reach the "final point of collapse". This final point of collapse is the point where the last pollinator collapses to extinction (as indicated in figure 2). Similarly, we can measure the ease of recovery by measuring the value of the driver of pollinator decline, where the first pollinator recovers from extinction. This would be the "first point of recovery" (as indicated in figure 3). The points of collapse and recovery as they were found for a certain value of connectance and nestedness are plotted in figure 5. For each value of connectance and nestedness, multiple networks were tested.

Connectance and nestedness both postpone the final point of collapse. Consequently, the persistence of the pollinator community to an increase in the driver of pollinator decline, $d_{A}$, increases with connectance and/or nestedness. Highly connected, and/or nested communities also recover from a collapse at higher values of the driver of pollinator decline. The distance between the final point of collapse and the first point of recovery, however, increases with connectance and/or nestedness. This means that a larger change in the driver of pollinator decline is needed for pollinators to recover, after the final threshold is passed. 
The net effect of species on each other. Our results show that the connectance and/or nestedness of mutualistic networks affects the stability of pollinator communities in various ways. The different aspects of stability discussed so far are the fraction of networks in which feasible solutions are found, the number of collapses and persistence of pollinator populations when the driver of pollinator decline, $d_{A}$, is increased, and the ease of recovery when the driver of pollinator decline, $d_{A}$, is decreased. Fortunately, these very different implications of network topology can all be understood when studying the "net effects" of species on each other.

Pollinators have a direct negative effect on each other due to competition. An indirect positive effect between pollinators may however occur when pollinator species interact with the same plant species. It is the interplay between these direct and indirect effects that ultimately determines the net effect of pollinators on each other (Bastolla et al. 2009). In figure 6, two pollinators interacting with the same plant species are shown to have an increasingly strong positive effect on each other. Not surprisingly, these pollinators can endure a larger increase in the driver of pollinator decline, $d_{A}$, than the pollinator not benefiting from this facilitation (also shown in figure 6). Once the tipping point is reached, the two pollinators interacting with the same plant species, however, collapse simultaneously, because they both depend on the same plant species.

Increased connectance and nestedness both increase the fraction of mutualistic partners shared by pollinators. The behaviour of highly connected, and/or highly nested communities, is therefore similar to the behaviour of the two pollinator species who share an interaction with the same plant species (see figure 6). With increasing connectance the "overlap" in identity of the mutualistic partners of pollinators is simply increased because a larger number of interactions has to be distributed over an equal number of plant species. The "rich get richer" mechanism that lies at the basis of the algorithm we used to generate nested networks, makes pollinators interact with mutualistic partners where many other pollinators already interact with. With the algorithm we thus achieve a similar increase in overlap while maintaining the number of interactions equal. As with the two species sharing an interaction with the same mutualistic partner in figure 6, pollinators who form part of a nested and/or highly connected community indirectly support each other when stress levels are high. This makes the community survive higher levels of the driver of pollinator decline, $d_{A}$, but also leads to a simultaneous collapse, because species depend on each other when stress levels are high.

Feasible solutions can be found in two types of regimes. The first regime would be one in which the combined effect of direct and indirect effects between pollinators is positive. An alternative regime is one where these net effects are mostly negative. This second regime is only feasible when these negative effects are relatively equal in strength. With increasing nestedness we move from the second to the first regime. Intermediate values of nestedness might be less likely to be in either of the two regimes. Some species have already benefited from the increase in nestedness, while others have not, which leads to an 
286 unbalanced community. This may explain why the probability of finding a feasible solution is smallest ${ }_{287}$ for intermediate values of nestedness (see figure 4 and supplementary material 3). 


\section{DISCUSSION}

Studies addressing the occurrence of critical transitions between alternative stable states in ecosystems have provided us with myriad examples of potential positive feedback mechanisms that might lay at the basis of them (May 1977; Scheffer et al. 2001; Scheffer \& Carpenter 2003; Rietkerk et al. 2004; Kefi et al. 2007; Hirota et al. 2011). These positive feedback mechanisms propel change towards an alternative stable state when environmental conditions pass a critical point (e.g., when a decline in population size reduces the growth of a population). It has, however, been challenging to understand how such mechanisms may affect the response of structurally complex systems, such as plant-pollinator communities, to changing environmental conditions (Scheffer et al. 2012). In this paper, we try to address this challenge by merging theory on alternative stable states with theory on the structure of ecological networks. Specifically, we show that pollinator populations may collapse suddenly to extinction, due to a positive feedback mechanism that results from the positive interactions between plants and pollinators. Each pollinator population described with our model is engaged in a unique positive feedback mechanism, of which the strength may vary substantially. Here, we show that such local positive feedback mechanisms may nonetheless provide the potential for a single community-wide collapse of pollinator populations, depending on the topology of mutualistic networks.

Our results can be understood intuitively by considering the "net effects" of species on each other and the way in which these effects are mediated by the topology of mutualistic networks. Pollinators have a direct negative effect on each other due to competition, while indirect positive effects may occur between pollinator species who interact with the same plant species. The extent to which pollinators interact with the same plant species increases with connectance and/or nestedeness. A high nestedness of the mutualistic network may therefore promote the occurrence of indirect positive effects between pollinators. Earlier work has shown that these indirect positive effects may reduce the effective competition between pollinators, and promote the coexistence of species in nested communities (Bastolla et al. 2009).

In this study, we show that the relative strength of indirect facilitation between pollinators becomes stronger as the driver of pollinator decline, $d_{A}$, increases (see figure 6 ). This corresponds to the increasingly popular 'stress-gradient hypothesis' which suggests that facilitative effects grow in importance as environmental stress increases (Bertness \& Callaway 1994; Holmgren et al. 1997; He et al. 2013). A high nestedness of mutualistic networks may therefore not only minimize effective competition to a level required for species coexistence; under stressful conditions, it may even promote strong indirect facilitation between pollinators.

We found that pollinators who are part of highly connected and/or nested communities can maintain themselves substantially longer than pollinators who are part of communities with a low nestedness as the driver of pollinator decline, $d_{A}$, is increased. This large persistence of pollinator populations under 
increasingly stressful conditions is, most likely, the result of the aforementioned indirect facilitation. Pollinator species who are part of either a highly nested or highly connected community can maintain themselves under stressful conditions because they indirectly support each other.

On the other hand, when species can survive under stressful conditions because they indirectly support each other, they also increasingly depend on each other as conditions get more stressful. As a consequence, pollinators collapse simultaneously once the driver of pollinator decline, $d_{A}$, passes a critical point. What we see in our model is therefore a surprising relationship between the capacity of species to coexist, to survive under stressful conditions, and the risk for a single community-wide collapse. They are all the result of the indirect positive effects, which are promoted by a high connectance and/or nestedness of mutualistic networks. Importantly, once collapsed, highly connected and/or nested communities may not necessarily recover more easily. In fact, our model shows the contrary. Recovery of pollinator populations who form part of highly nested communities require a quite large decrease in the driver of pollinator decline, $d_{A}$, in comparison to pollinator populations who form part of communities with a low nestedness.

Our findings may have large implications for our view on the sustainability of natural communities and the ecosystem services provided by them. Based on the insurance hypothesis, one expects ecosystems services to be more reliable when supported by a large number of species (Naeem \& Li 1997; Yachi \& Loreau 1999). Functional redundancy of species is often seen as a valuable 'commodity', because it makes ecosystems more reliable in terms of the ecosystem services they provide (see Naeem \& Li 1997). Our analysis, however, illustrates that the functional overlap of pollinators, which is related to the connectivity and/or nestedness of mutualistic networks, may simultaneously increase the risk for a single community-wide collapse. A valuable ecosystem service, namely pollination, can therefore be lost suddenly, despite the fact that it is provided by a large number of species who are, when taking only their intrinsic properties into account, not equally sensitive to the driver of pollinator decline, $d_{A}$.

Our study is one of many small steps needed to bring theory on critical transitions and the structure of ecological networks together and we realize that this paper raises new questions that require further exploration. First, even though our model is substantially more complex than many others that study critical transitions, it is constrained to mutualistically interacting plant-pollinator communities. Multiple types of interactions co-occur in natural communities (Melián et al. 2009), and future studies should explore how the structuring of multiple types of interactions affect critical transitions. Secondly, our results underline the importance of developing early-warning signals for critical transitions in ecological networks (Scheffer et al. 2009). Third and finally, as the mechanisms we describe are generic, it is possible that a similar trade-off between persistence under severe conditions and potential for a systemic collapse 
occurs in other systems as well. This is reinforced by previous studies finding notable similarities between the structure of mutualistic networks and that of financial systems (Uzzi 1996; May et al. 2008; Saavedra et al. 2008; Haldane \& May 2011; Saavedra et al. 2011).

\section{ACKNOWLEDGEMENTS}

We thank Kate O'Brien and three anonymous referees for their valuable suggestions and comments. This work was partly funded by a Netherlands Organization for Scientific Research (NWO) Spinoza Prize, awarded to Marten Scheffer, and two European Research Council (ERC) Advanced Grants awarded to Marten Scheffer and Jordi Bascompte. 


\section{REFERENCES}

Allen-Wardell, G., Bernhardt, P., Bitner, R., Burquez, A., Buchmann, S., Cane, J. et al. (1998). The Potential Consequences of Pollinator Declines on the Conservation of Biodiversity and Stability of Food Crop Yields. Conserv. Biol., 12, 1, 8-17.

Bascompte, J. \& Jordano, P. (2007). Plant-animal mutualistic networks: The architecture of biodiversity. Annu. Rev. Ecol. Evol. Syst., 38, 567-593.

Bascompte, J., Jordano, P., Melián, C. J. \& Olesen, J. M. (2003). The nested assembly of plant-animal mutualistic networks. Proc. Natl. Acad. Sci. U.S.A., 100, 16, 9383-9387.

Bascompte, J., Jordano, P. \& Olesen, J. M. (2006). Asymmetric coevolutionary networks facilitate biodiversity maintenance. Science, 312, 5772, 431-433.

Bastolla, U., Fortuna, M. A., Pascual-Garcia, A., Ferrera, A., Luque, B. \& Bascompte, J. (2009). The architecture of mutualistic networks minimizes competition and increases biodiversity. Nature, 458, 7241, 1018-U91.

Bertness, M. D. \& Callaway, R. (1994). Positive interactions in communities. Trends in Ecology \&3 Evolution, 9, 5, 191-193.

Biesmeijer, J., Roberts, S., Reemer, M., Ohlemüller, R., Edwards, M., Peeters, T. et al. (2006). Parallel declines in pollinators and insect-pollinated plants in britain and the netherlands. Science, 313, 5785, 351.

Bryden, J., Gill, R. J., Mitton, R. A., Raine, N. E. \& Jansen, V. A. (2013). Chronic sublethal stress causes bee colony failure. Ecology letters, 16, 12, 1463-1469.

Burgos, E., Ceva, H., Perazzo, R. P., Devoto, M., Medan, D., Zimmermann, M. et al. (2007). Why nestedness in mutualistic networks? J. Theor. Biol., 249, 2, 307-313.

Burkle, L. A., Marlin, J. C. \& Knight, T. M. (2013). Plant-pollinator interactions over 120 years: Loss of species, co-occurrence and function. Science.

Dai, L., Vorselen, D., Korolev, K. S. \& Gore, J. (2012). Generic indicators for loss of resilience before a tipping point leading to population collapse. Science, 336, 6085, 1175-1177.

Diaz, S., Chapin III, F. \& Potts, S. (2005). Biodiversity regulation of ecosystem services. Ecosystems and human well-being: current state and trends: findings of the Condition and Trends Working Group of the Millennium Ecosystem Assessment, 1, 297.

Drake, J. M. \& Griffen, B. D. (2010). Early warning signals of extinction in deteriorating environments. Nature, 467, 7314, 456-459. 
Fortuna, M. A. \& Bascompte, J. (2006). Habitat loss and the structure of plant-animal mutualistic networks. Ecol. Lett., 9, 278-283.

Garibaldi, L. A., Steffan-Dewenter, I., Winfree, R., Aizen, M. A., Bommarco, R., Cunningham, S. A. et al. (2013). Wild pollinators enhance fruit set of crops regardless of honey bee abundance. Science.

Haldane, A. G. \& May, R. M. (2011). Systemic risk in banking ecosystems. Nature, 469, 7330, 351-355.

He, Q., Bertness, M. D. \& Altieri, A. H. (2013). Global shifts towards positive species interactions with increasing environmental stress. Ecology letters.

Henry, M., Beguin, M., Requier, F., Rollin, O., Odoux, J.-F., Aupinel, P. et al. (2012). A common pesticide decreases foraging success and survival in honey bees. Science.

Hirota, M., Holmgren, M., Van Nes, E. \& Scheffer, M. (2011). Global resilience of tropical forest and savanna to critical transitions. Science, 334, 6053, 232-235.

Holmgren, M., Scheffer, M. \& Huston, M. A. (1997). The interplay of facilitation and competition in plant communities. Ecology, 78, 7, 1966-1975.

Ives, A. R. \& Carpenter, S. R. (2007). Stability and diversity of ecosystems. Science, 317, 5834, 58-62.

Jordano, P., Bascompte, J. \& Olesen, J. (2003). Invariant properties in coevolutionary networks of plant-animal interactions. Ecol. Lett., 6, 1, 69-81.

Kefi, S., S., Rietkerk, M., Alados, C., Pueyo, Y., Papanastasis, V., ElAich, A. et al. (2007). Spatial vegetation patterns and imminent desertification in mediterranean arid ecosystems. Nature, 449, 7159, 213-217.

Klein, A., Vaissiere, B., Cane, J., Steffan-Dewenter, I., Cunningham, S., Kremen, C. et al. (2007). Importance of pollinators in changing landscapes for world crops. Proc. R. Soc. London, Ser. B, 274, $1608,303$.

May, R. (1977). Thresholds and breakpoints in ecosystems with a multiplicity of stable states. Nature, $269,5628,471-477$.

May, R., Levin, S. \& Sugihara, G. (2008). Complex systems: ecology for bankers. Nature, 451, 7181, 893-895.

May, R. M. (1972). Will a large complex system be stable. Nature, 238, 5364, 413-\&.

May, R. M. (2006). Network structure and the biology of populations. Trends Ecol. Evol., 21, 7, 394-399.

McCann, K. S. (2000). The diversity-stability debate. Nature, 405, 6783, 228-233. 
Medan, D., Perazzo, R. P., Devoto, M., Burgos, E., Zimmermann, M. G., Ceva, H. et al. (2007). Analysis and assembling of network structure in mutualistic systems. J. Theor. Biol., 246, 510-512.

Melián, C. J., Bascompte, J., Jordano, P. \& Krivan, V. (2009). Diversity in a complex ecological network with two interaction types. Oikos, 118, 1, 122-130.

Memmott, J., Waser, N. M. \& Price, M. V. (2004). Tolerance of pollination networks to species extinctions. Proc. R. Soc. London, Ser. B, 271, 1557, 2605-2611.

Naeem, S. \& Li, S. (1997). Biodiversity enhances ecosystem reliability. Nature, 390, 6659, 507-509.

Okuyama, T. \& Holland, J. N. (2008). Network structural properties mediate the stability of mutualistic communities. Ecol. Lett., 11, 3, 208-216.

Ollerton, J., Winfree, R. \& Tarrant, S. (2011). How many flowering plants are pollinated by animals? Oikos, 120, 3, 321-326.

Potts, S., Biesmeijer, J., Kremen, C., Neumann, P., Schweiger, O. \& Kunin, W. (2010). Global pollinator declines: trends, impacts and drivers. Trends Ecol. Evol., 25, 6, 345-353.

Rietkerk, M., Dekker, S. C., de Ruiter, P. C. \& van de Koppel, J. (2004). Self-organized patchiness and catastrophic shifts in ecosystems. Science, 305, 5692, 1926-1929.

Saavedra, S., Reed-Tsochas, F. \& Uzzi, B. (2008). A simple model of bipartite cooperation for ecological and organizational networks. Nature, 457, 7228, 463-466.

Saavedra, S., Stouffer, D., Uzzi, B. \& Bascompte, J. (2011). Strong contributors to network persistence are the most vulnerable to extinction. Nature.

Scheffer, M., Bascompte, J., Brock, W. A., Brovkin, V., Carpenter, S. R., Dakos, V. et al. (2009). Early-warning signals for critical transitions. Nature, 461, 7260, 53-59.

Scheffer, M., Carpenter, S., Foley, J. A., Folke, C. \& Walker, B. (2001). Catastrophic shifts in ecosystems. Nature, 413, 6856, 591-596.

Scheffer, M. \& Carpenter, S. R. (2003). Catastrophic regime shifts in ecosystems: linking theory to observation. Trends in Ecology \& Evolution, 18, 12, 648-656.

Scheffer, M., Carpenter, S. R., Lenton, T. M., Bascompte, J., Brock, W., Dakos, V. et al. (2012). Anticipating critical transitions. Science, 338, 6105, 344-348.

Stone, L. \& Roberts, A. (1991). Conditions for a species to gain advantage from the presence of competitors. Ecology, pp. 1964-1972. 
Thébault, E. \& Fontaine, C. (2010). Stability of ecological communities and the architecture of mutualistic and trophic networks. Science, 329, 5993, 853-856.

Uzzi, B. (1996). The sources and consequences of embeddedness for the economic performance of organizations: The network effect. Am. Sociol. Rev., pp. 674-698.

Van Nes, E. H. \& Scheffer, M. (2004). Large species shifts triggered by small forces. Am. Nat., 164, 2, $255-266$.

Veraart, A. J., Faassen, E. J., Dakos, V., van Nes, E. H., Lürling, M. \& Scheffer, M. (2011). Recovery rates reflect distance to a tipping point in a living system. Nature, 481, 7381, 357-359.

Whitehorn, P. R., O’Connor, S., Wackers, F. L. \& Goulson, D. (2012). Neonicotinoid pesticide reduces bumble bee colony growth and queen production. Science.

Yachi, S. \& Loreau, M. (1999). Biodiversity and ecosystem productivity in a fluctuating environment: The insurance hypothesis. Proc. Natl. Acad. Sci. U.S.A., 96, 4, 1463-1468. 


\section{FIGURE CAPTIONS}

Figure 1: Matrix representations of a randomly structured network (left) and a nested network (right, $\mathrm{N}=0.6$ ). Filled squares indicate interactions between species. Column and row numbers correspond to individual plant and pollinator species. Species are ordered based upon their number of interactions.

Figure 2: The collapse of pollinator populations when the driver of pollinator decline, $d_{A}$, affecting growth and/or mortality of pollinators, is gradually increased from zero to one. Results are shown for a random (left) and a nested (right, $\mathrm{N}=0.6)$ network. Connectance of both networks is equal $(\mathrm{D}=0.15)$. Several extinction events precede the final collapse of the randomly structured plant-pollinator community, while the nested community exhibits only one point of community-wide collapse.

Figure 3: The recovery of pollinator populations when the driver of pollinator decline, $d_{A}$, is gradually decreased from one to zero. The points of recovery are not necessarily equal to the points of collapse (see figure 2). Especially in the nested community a large difference is observed between the final point of collapse and the first point of recovery. A substantial reduction of the driver of pollinator decline might thus be necessary for pollinator populations to recover from a collapse.

Figure 4: The number of collapses observed in randomly structured communities with different levels of connectance (left), and in communities with increasingly nested network topologies with a fixed connectance of 0.15 and fraction of forbidden links of 0.3 (right). The coloured bars represent the fraction of feasible networks in which a certain number of collapses is found. The fraction of networks in which feasible solutions are found is indicated with the green diamonds.

Figure 5: Points of collapse (circles) when the driver of pollinator decline, $d_{A}$, is increased, and points of recovery (triangles) when the driver of pollinator decline, $d_{A}$, is decreased. As in figure 4 , results are shown for randomly structured networks that vary in connectance (left), and for increasingly nested networks with a connectance of 0.15 and fraction of forbidden links of 0.3 (right). In case of multiple collapses and/or recoveries, the final point of collapse and the first point of recovery was plotted. 
Figure 6: The net effect of species on each other while the driver of pollinator decline increases. Pollinators that share a mutualistic partner have an increasingly positive effect on each other and collapse simultaneously. Pollinators that do not share mutualistic partners have an increasingly negative effect on each other and collapse independently. A. A simple network of mutualistic interactions between plants and pollinators. Pollinator A1 and A2 share mutualistic partner P1, while pollinator A3 does not share its mutualistic partner P2. Dashed lines indicate net-relationships between pollinators. Although pollinators are in direct competition with each other, net-positive relationships may exist between pollinator A1 and A2. B. Net effect $\left(d A_{k} / d r_{l}\right)$ of pollinator species on each other. In blue the net effects of pollinators A1 and A2 on each other. In green the net-relationships between pollinator A3 and the other two pollinators. C. Abundance of pollinators A1 and A2 (blue), and pollinator A3 (red). 
Random network structure

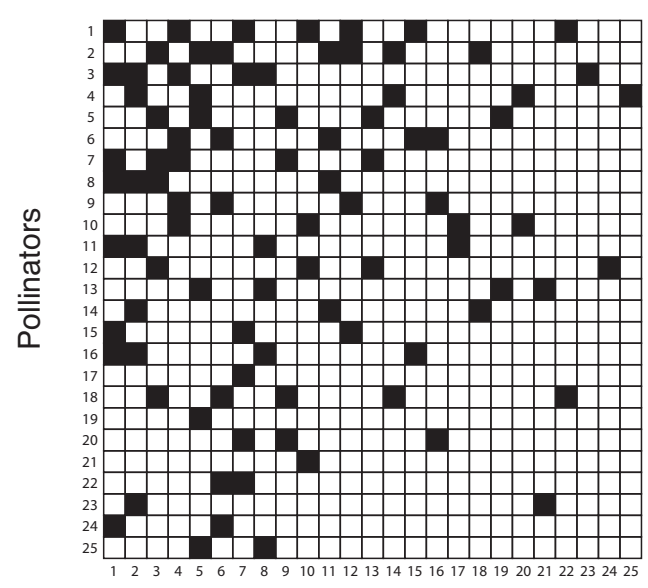

Plants
Nested network structure

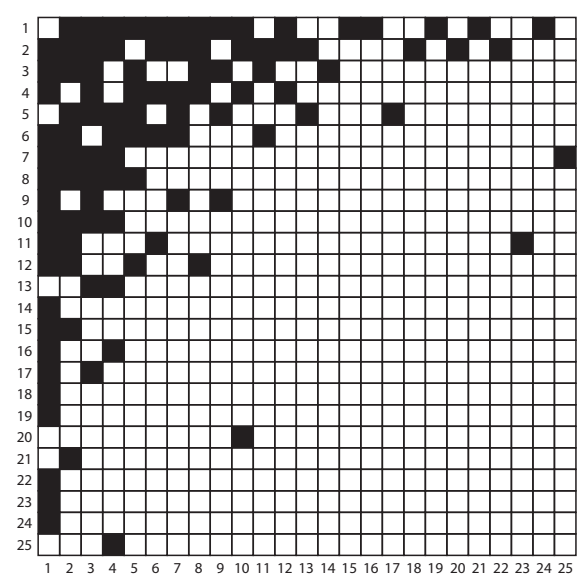

Plants

Figure 1: Matrix representations of a randomly structured network (left) and a nested network (right, $\mathrm{N}=0.6$ ). Filled squares indicate interactions between species. Column and row numbers correspond to individual plant and animal species. Species are ordered based upon their number of interactions. 
Random network structure

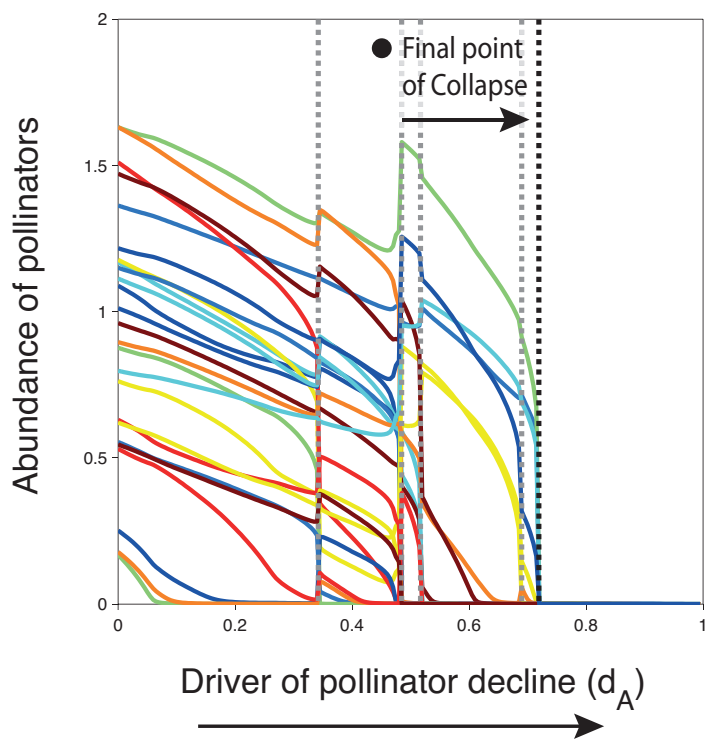

Nested network structure

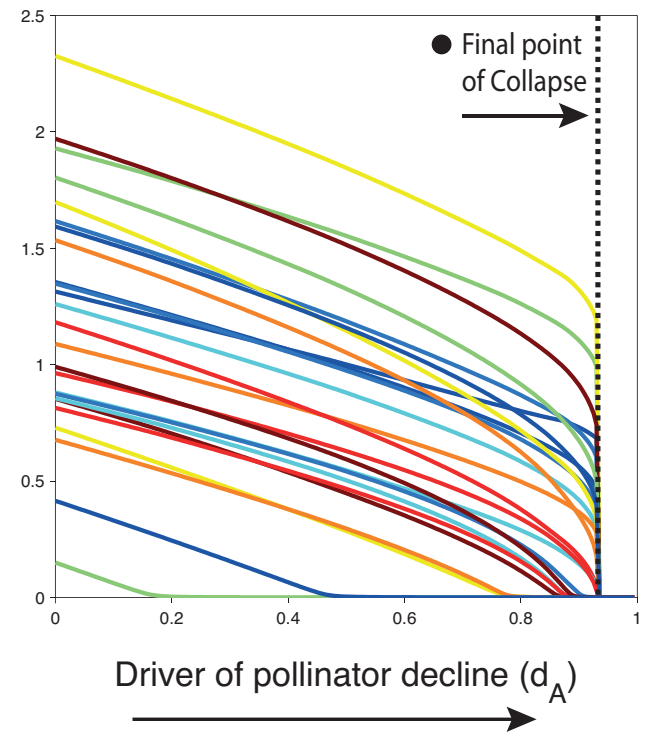

Figure 2: The collapse of pollinator populations when the driver of pollinator decline, $d_{A}$, affecting growth and/or mortality of pollinators, is gradually increased from zero to one. Results are shown for a random (left) and a nested (right, $\mathrm{N}=0.6)$ network. Connectance of both networks is equal $(\mathrm{D}=0.15)$. Several extinction events precede the final collapse of the randomly structured plant-pollinator community, while the nested community exhibits only one point of community-wide collapse.
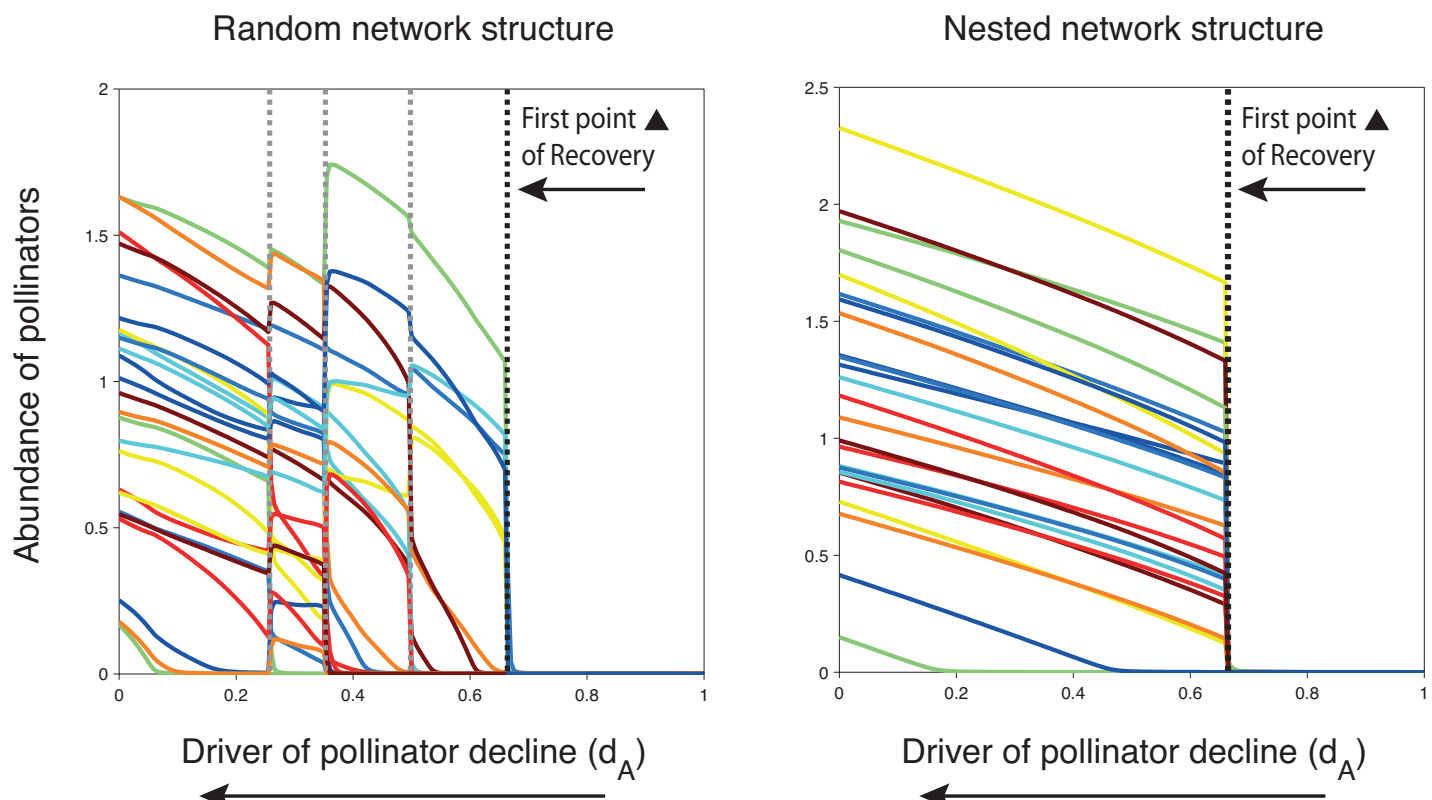

Figure 3: The recovery of pollinator populations when the driver of pollinator decline, $d_{A}$, is gradually decreased from one to zero. The points of recovery are not necessarily equal to the points of collapse (see figure 2). Especially in the nested community a large difference is observed between the final point of collapse and the first point of recovery. A substantial reduction of the driver of pollinator decline might thus be necessary for pollinator populations to recover from a collapse. 


\section{Random network structure}

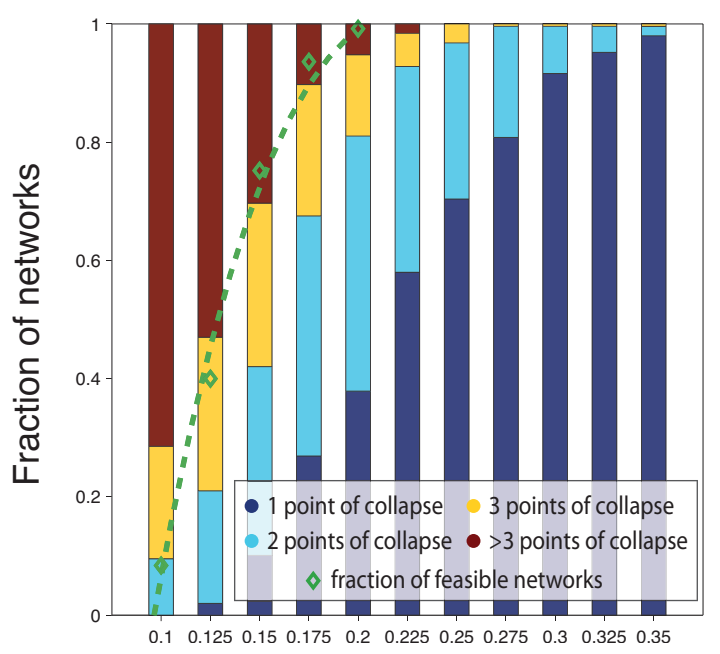

Connectance
Increasingly nested structure

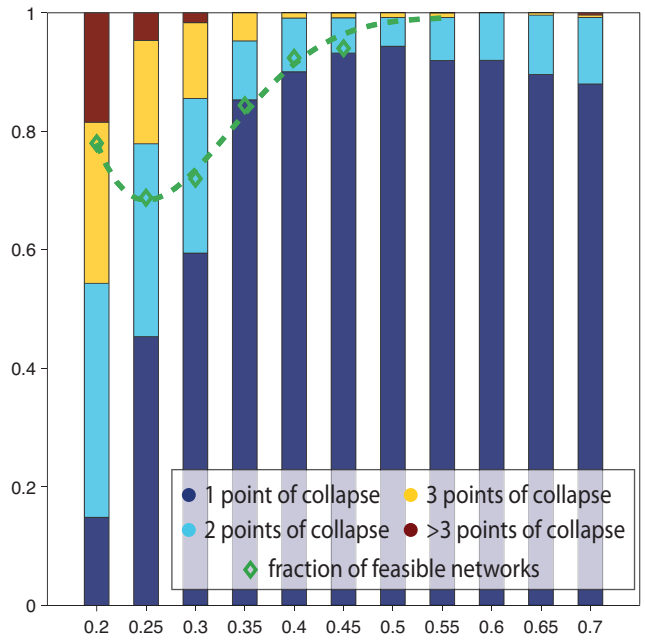

Nestedness

Figure 4: The number of collapses observed in randomly structured communities with different levels of connectance (left), and in communities with increasingly nested network topologies with a fixed connectance of 0.15 and fraction of forbidden links of 0.3 (right). The coloured bars represent the fraction of feasible networks in which a certain number of collapses is found. The fraction of networks in which feasible solutions are found is indicated with the green diamonds.
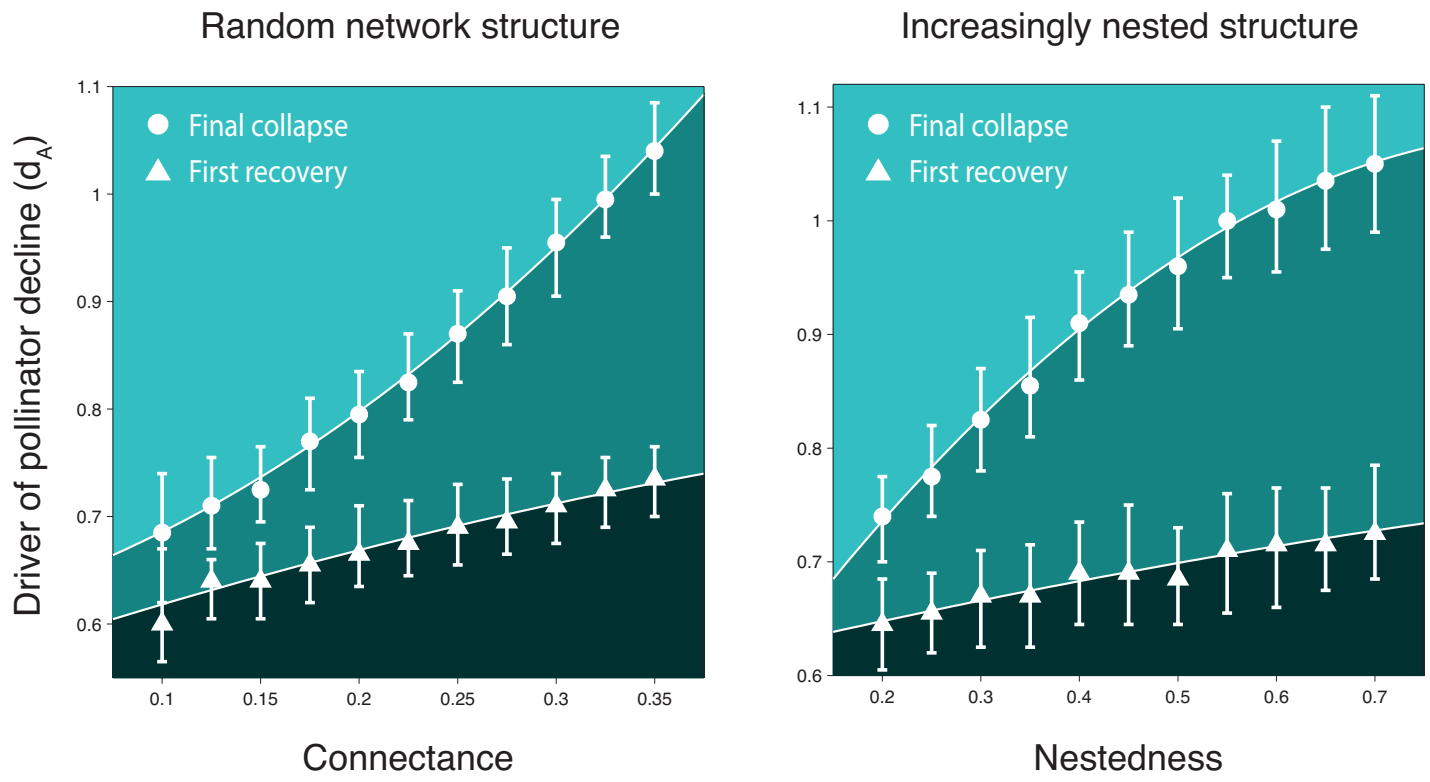

Figure 5: Points of collapse (circles) when the driver of pollinator decline, $d_{A}$, is increased, and points of recovery (triangles) when the driver of pollinator decline, $d_{A}$, is decreased. As in figure 4 , results are shown for randomly structured networks that vary in connectance (left), and for increasingly nested networks with a connectance of 0.15 and fraction of forbidden links of 0.3 (right). In case of multiple collapses and/or recoveries, the final point of collapse and the first point of recovery was plotted. 
Figure 6. The net effect of species on each other while the driver of pollinator decline increases. Pollinators that share a mutualistic partner have an increasingly positive effect on each other and collapse simultaneously. Pollinators that do not share mutualistic partners have an increasingly negative effect on each other and collapse independently.

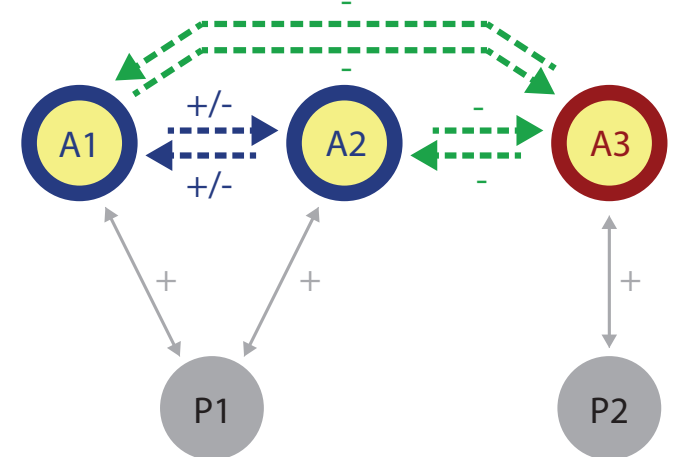

A. A simple network of mutualistic interactions between plants and pollinators. Pollinator A1 and A2 share mutualistic partner P1, while pollinator A3 does not share its mutualistic partner P2. Dashed lines indicate net-relationships between pollinators. Although pollinators are in direct competition with each other, net-positive relationships may exist between pollinator A1 and A2.

parameter settings: $r_{i} \sim U(0.15,0.25), \gamma_{0, i k} \sim U(0.9,1.1), t=0.5, h_{i} \sim U(0.5,0.6)$, $C_{i i} \sim U(0.4,0.6), C_{i j} \sim U(0.025,0.075)$, and $\mu=0.0001$.

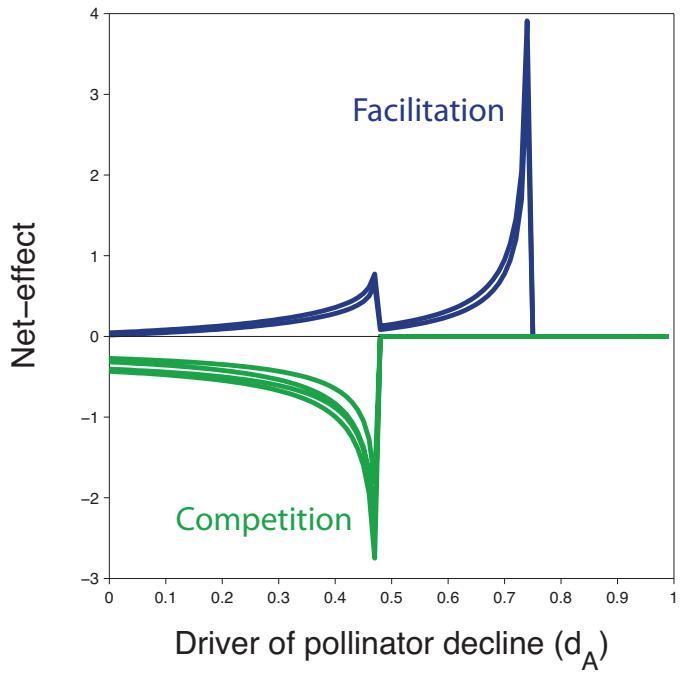

B. Net effect $\left(d A_{k} / d r_{l}\right)$ of pollinator species on each other. In blue the net effects of pollinators A1 and A2 on each other. In green the net-relationships between pollinator A3 and the other two pollinators

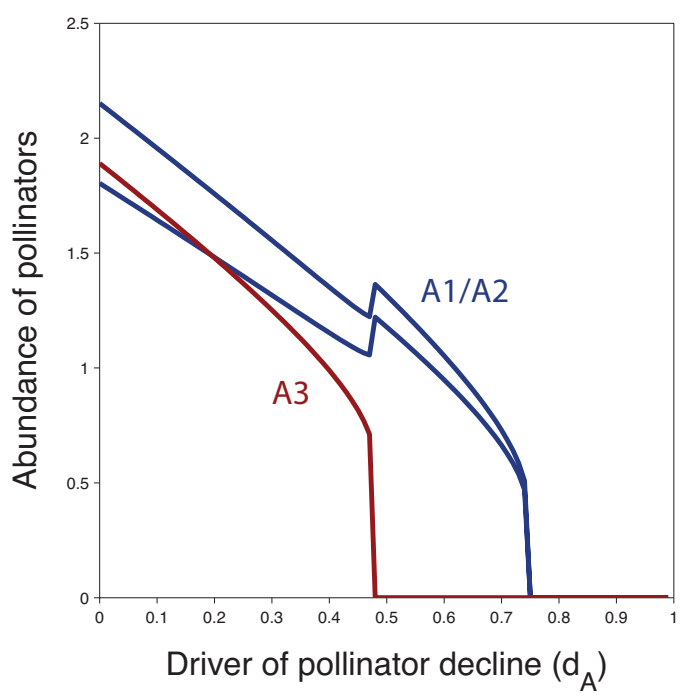

C. Abundance of pollinators A1 and A2 (blue), and pollinator A3 (red). 


\section{SUPPLEMENTARY MATERIAL 1}

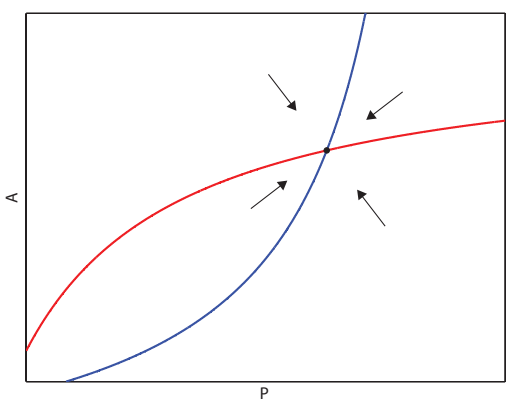

(a) $d_{A}<r_{A}$

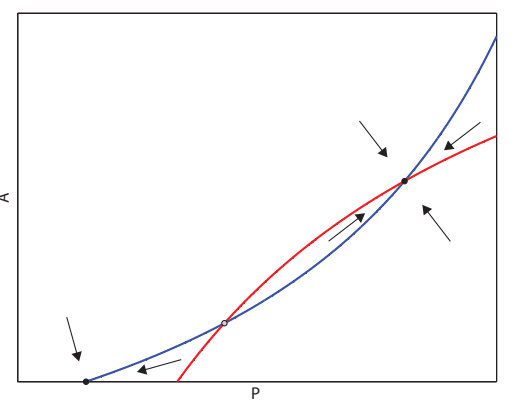

(b) $d_{A}>r_{A}$

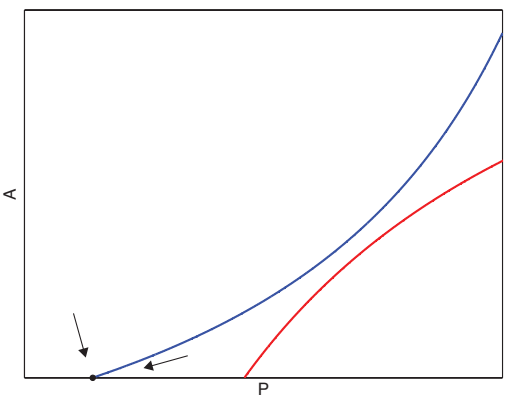

(c) $d_{A}>>r_{A}$

Figure S1: Nullclines of two mutualistically interacting species. Filled dots indicate stable equilibria, open dots indicate unstable equilibria. Fundamentally different configurations exist when (a) the driver of pollinator decline, $d_{A}$, is smaller than intrinsic growth rate $r_{A}$, (b) when the driver of pollinator decline, $d_{A}$, is bigger than intrinsic growth rate $r_{A}$ and, (c) when the driver of pollinator decline, $d_{A}$, is substantially larger than intrinsic growth rate $r_{A}$. By increasing the driver of pollinator decline, $d_{A}$, we change from a regime with one stable state, presented in $a$, to the regime with two alternative stable states presented in $b$, until eventually a tipping point is reached where pollinators collapse to extinction. For a further analysis of models with two mutualistically interacting species see May (1978), Dean (1983), and Wright (1989). 
Random network structure

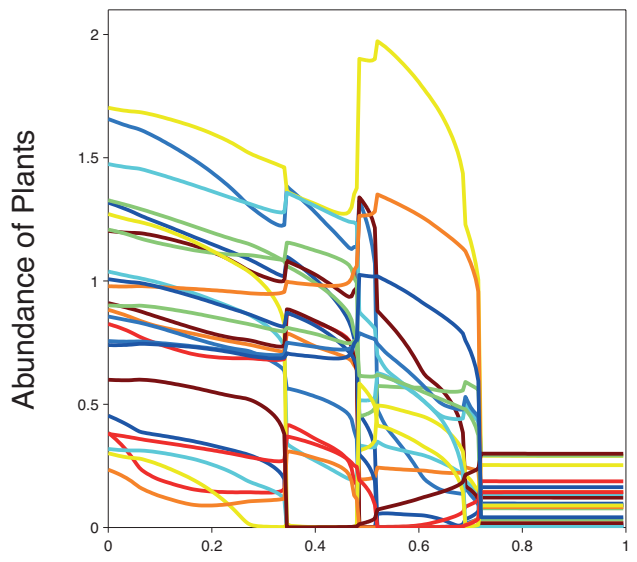

Driver of pollinator decline $\left(d_{A}\right)$
Nested network structure

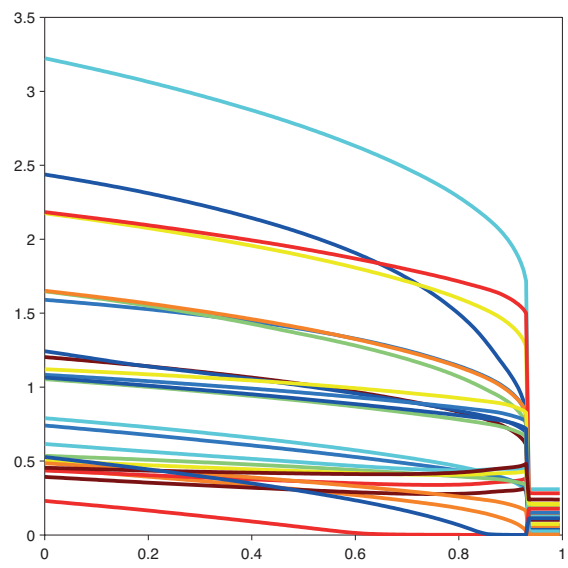

Driver of pollinator decline $\left(d_{A}\right)$

Figure S2: Collapse of plant populations when increasing the mortality $d_{A}$ of pollinators. Results are shown for a random (left) and a nested (right, $\mathrm{N}=0.6$ ) network. Parameter settings are the same as in figure 2 .
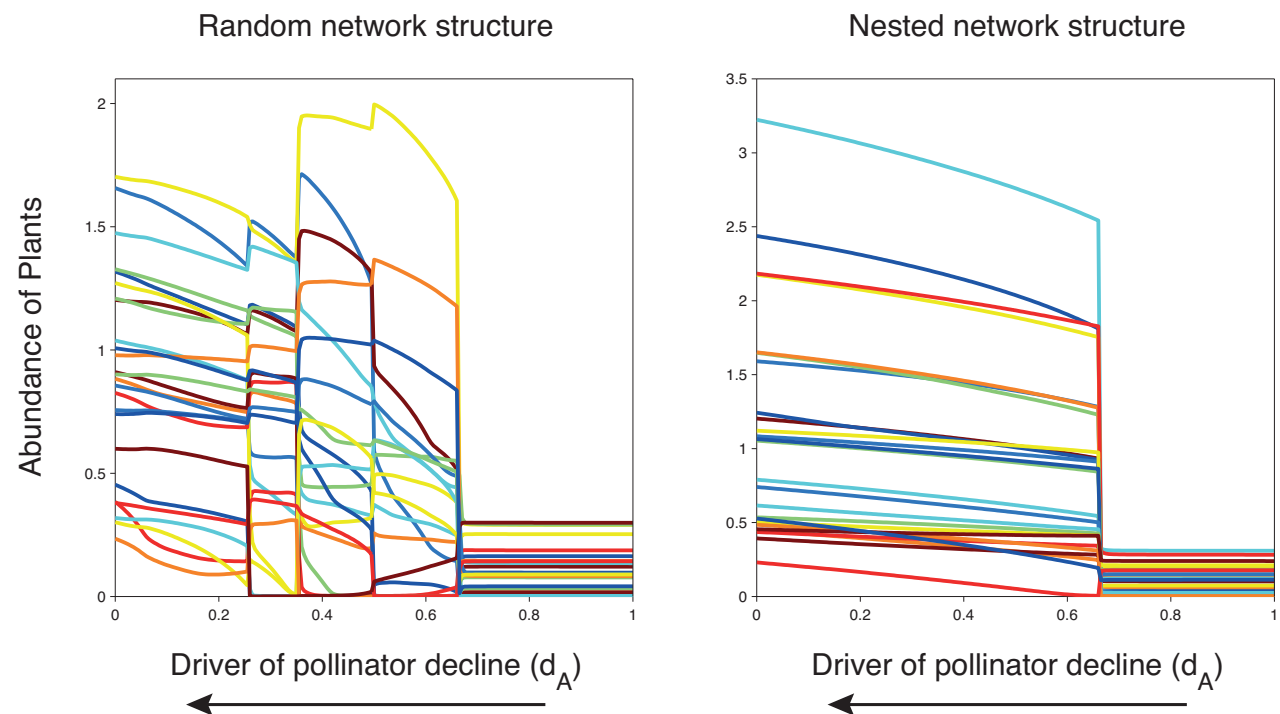

Figure S3: Re-establishment of plant populations when decreasing the mortality of pollinators $d_{A}$. Results are shown for a random (left) and a nested (right, $\mathrm{N}=0.6$ ) network. Parameter settings are the same as in figure 3. 


\section{SUPPLEMENTARY MATERIAL 3}

We tested the extent to which our results depend on the specific number of species, connectance or fraction of forbidden links chosen (see figure S6, S7, S4 and S5).

Furthermore, we show in figure S8 and S9 what our results look like if we do not allow any species to have less than 2 partners during any step of the algorithm we used to generate nested networks.

We only found qualitative differences in the behaviour of our model. 
Random network structure

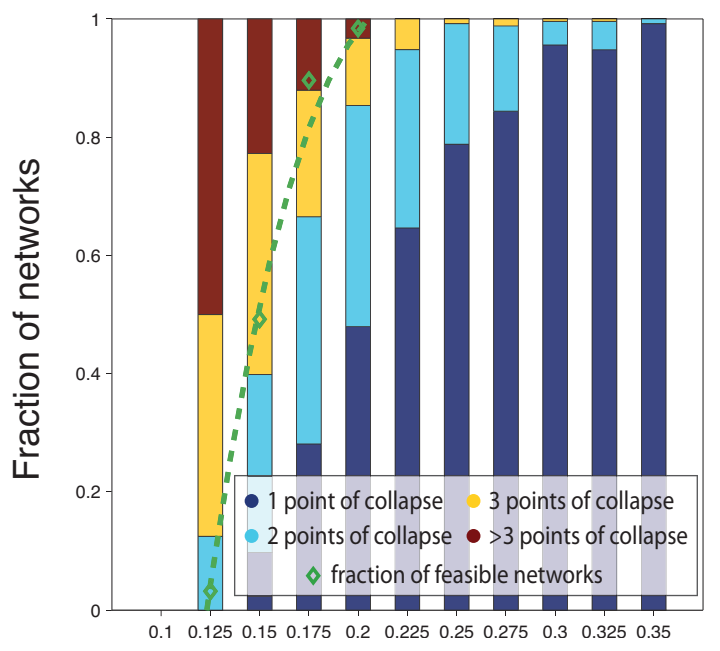

Connectance
Increasingly nested structure

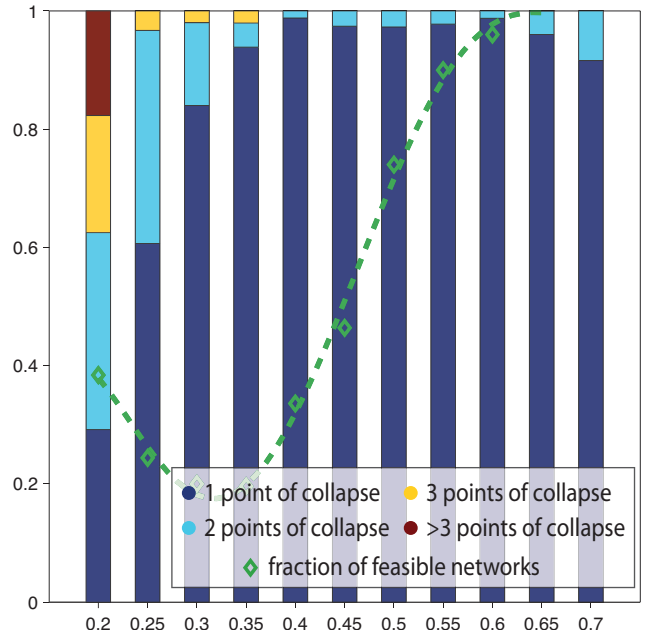

Nestedness

Figure S4: Results when using the same parameter settings as in figure 4, only now the community consists out of 35 plant and 35 pollinator species. As in figure 4 , the coloured bars represent the fractions of feasible networks in which a certain number of collapses is found. The fraction of networks in which feasible solutions are found is indicated with the green diamonds.
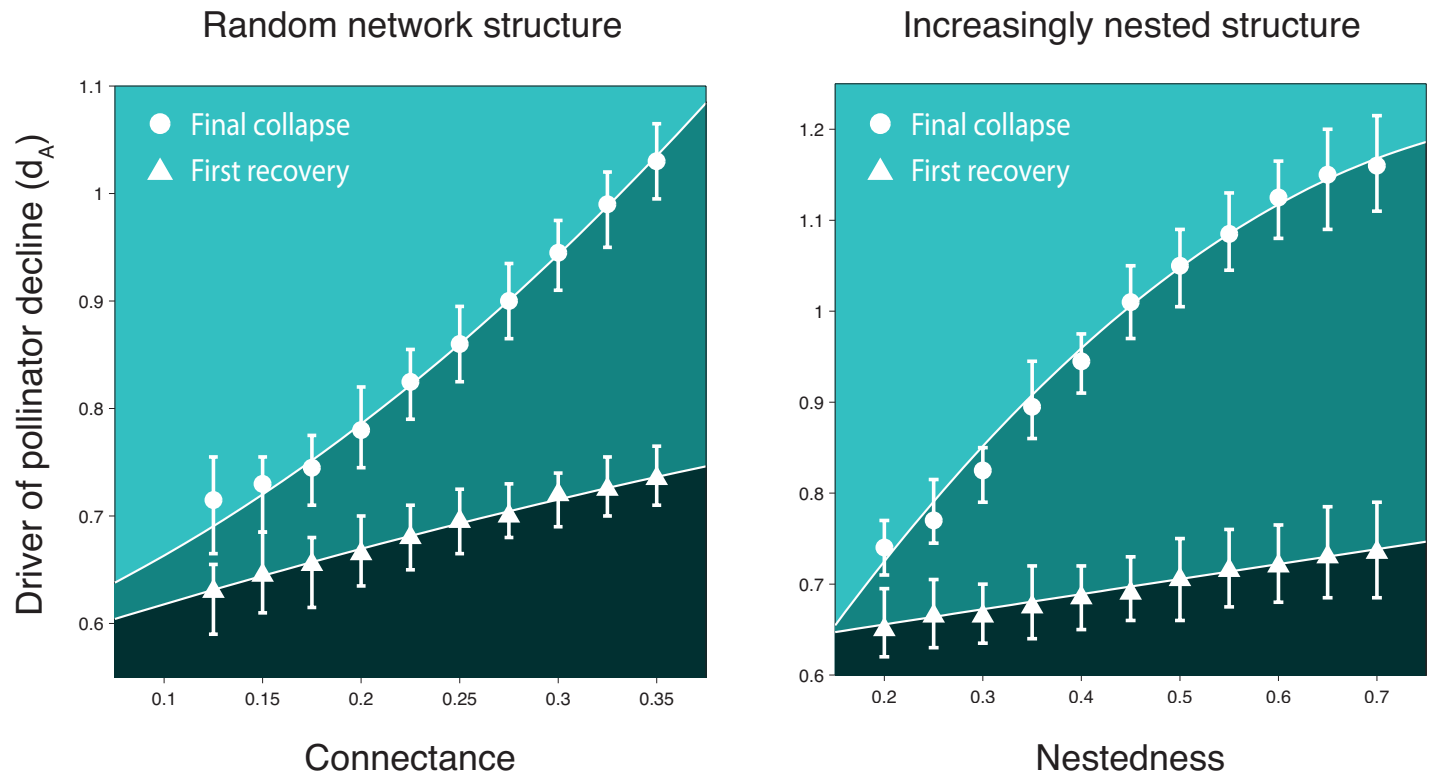

Figure S5: Points of collapse (circles) when the driver of pollinator decline, $d_{A}$, is increased, and points of recovery (triangles) when the driver of pollinator decline, $d_{A}$, is decreased. In case of multiple collapses and/or recoveries, the final point of collapse and the first point of recovery was plotted. Parameter settings are as in figure $\mathrm{S} 4$. 
Random network structure

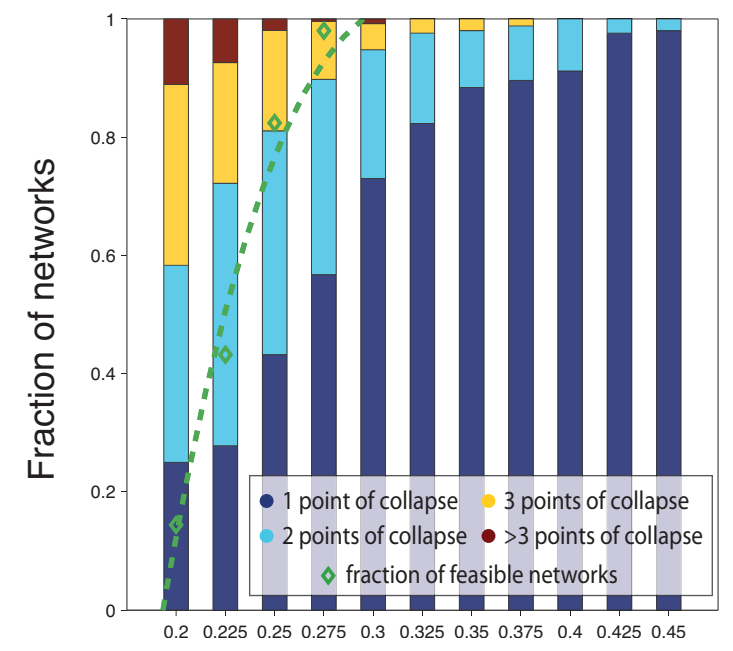

Connectance
Increasingly nested structure

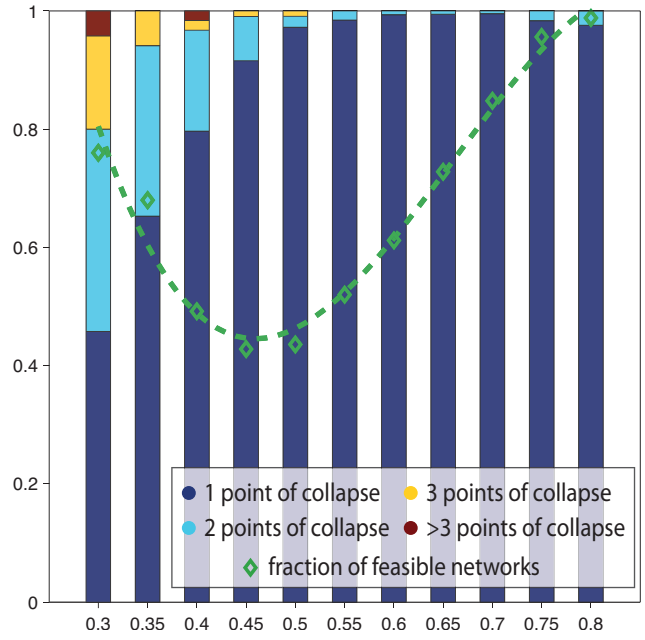

Nestedness

Figure S6: Results when using the same parameter settings as in figure 4, only now competition between species is a bit stronger, $C_{i j} \sim U(0.025,0.075)$, and in communities with increasingly nested network topologies (right panel), the connectance is fixed to 0.25 , and the fraction of forbidden links is fixed to 0.25 . As in figure 4 , the coloured bars represent the fractions of feasible networks in which a certain number of collapses is found. The fraction of networks in which feasible solutions are found is indicated with the green diamonds.

\section{Random network structure}

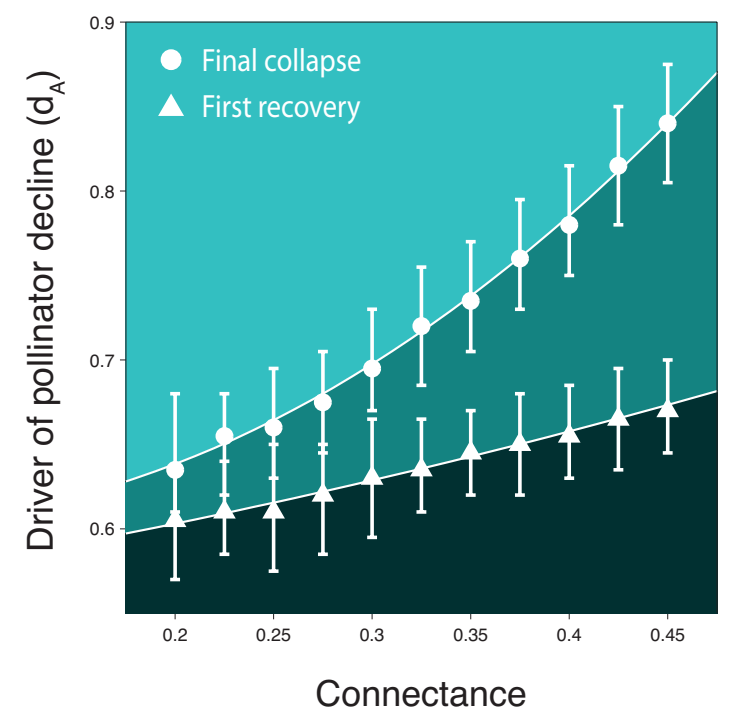

Increasingly nested structure

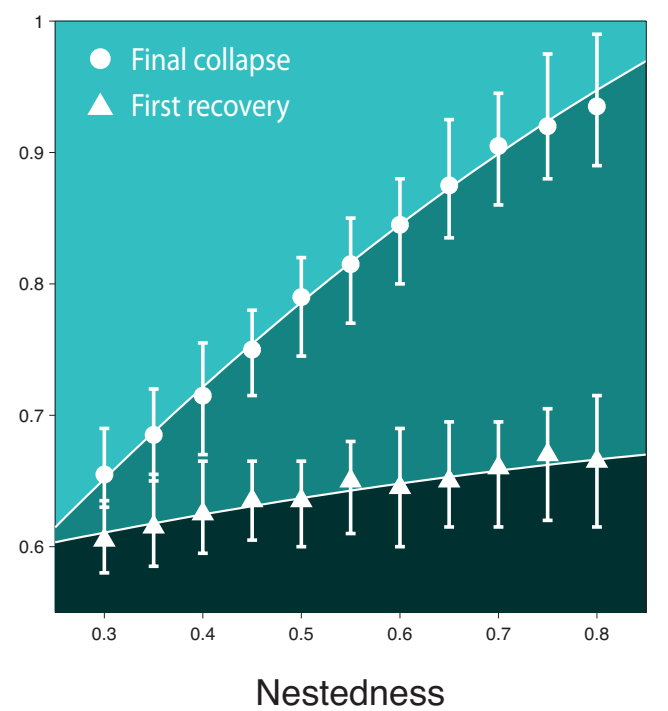

Figure S7: Points of collapse (circles) when the driver of pollinator decline, $d_{A}$, is increased, and points of recovery (triangles) when the driver of pollinator decline, $d_{A}$, is decreased. In case of multiple collapses and/or recoveries, the final point of collapse and the first point of recovery was plotted. Parameter settings are as in figure $\mathrm{S} 6$. 
Random network structure

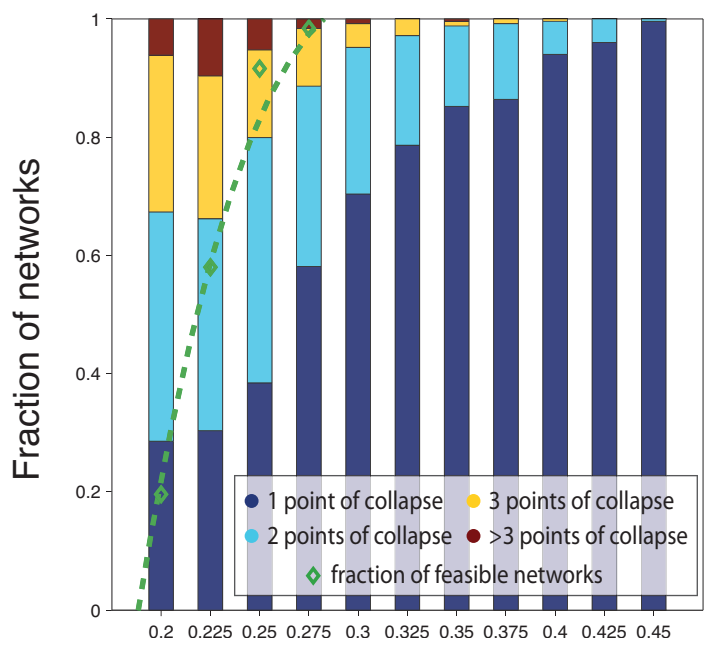

Connectance
Increasingly nested structure

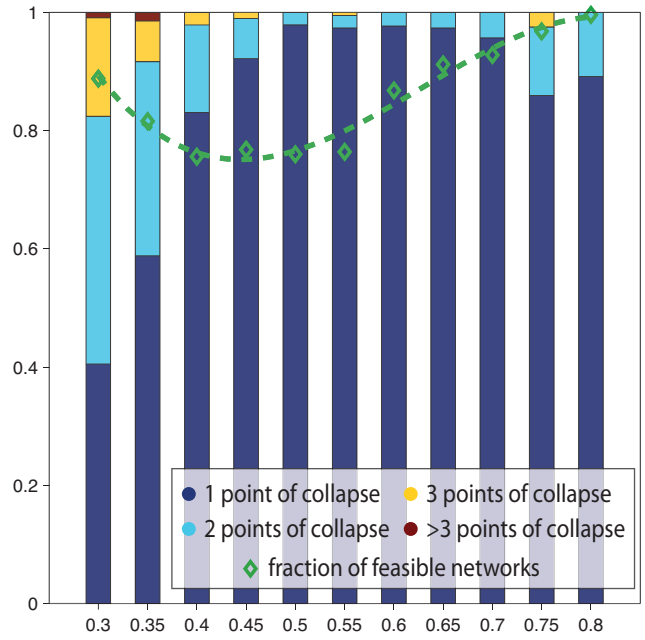

Nestedness

Figure S8: Results when using the same parameter settings as in figure S6, only now each species has at least two interactions. As in figure S6, the coloured bars represent the fractions of feasible networks in which a certain number of collapses is found. The fraction of networks in which feasible solutions are found is indicated with the green diamonds.
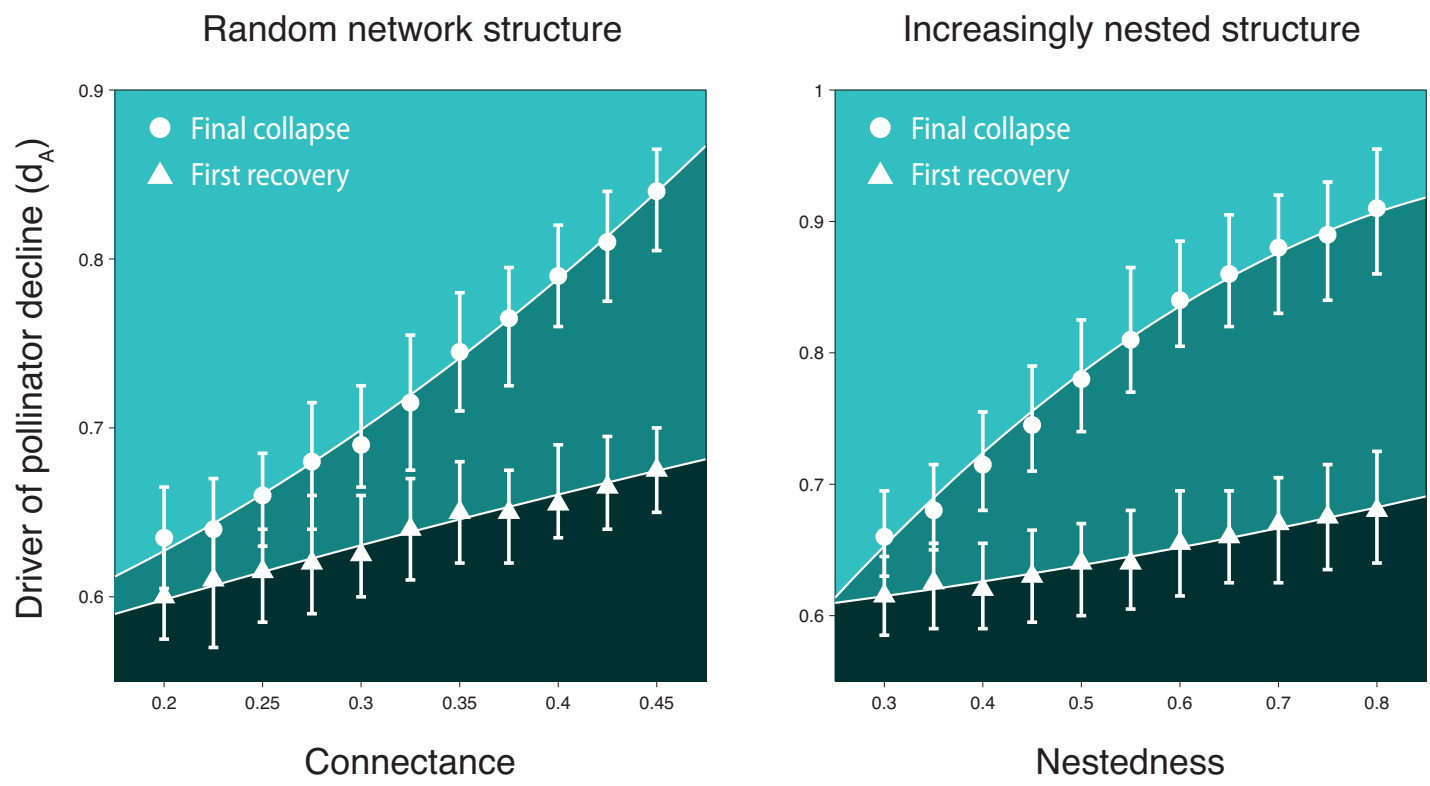

Figure S9: Points of collapse (circles) when the driver of pollinator decline, $d_{A}$, is increased, and points of recovery (triangles) when the driver of pollinator decline, $d_{A}$, is decreased. In case of multiple collapses and/or recoveries, the final point of collapse and the first point of recovery was plotted. Parameter settings are as in figure S8. 


\section{REFERENCES SUPPLEMENTARY MATERIAL}

Dean, A. M. (1983). A simple model of mutualism. Am. Nat., 121, 3, pp. 409-417.

May, R. (1978). Studies in Mathematical Ecology. II Populations and Communities, chapter Mathematical aspects of the dynamics of animal populations., pp. 317-366. Washington, DC:

Mathematical Association of America.

Wright, D. H. (1989). A simple, stable model of mutualism incorporating handling time. Am. Nat., 134, 4, pp. 664-667. 